\title{
Orbital migration and the frequency of giant planet formation
}

\author{
D. E. Trilling ${ }^{1}$, J. I. Lunine ${ }^{2}$, and W. Benz ${ }^{3}$ \\ ${ }^{1}$ University of Pennsylvania, Department of Physics and Astronomy, David Rittenhouse Laboratory, 209 S. 33rd St., \\ Philadelphia, PA 19104, USA \\ 2 Lunar and Planetary Laboratory, University of Arizona, Tucson, AZ 85721, USA \\ 3 Physikalisches Institut, Universitaet Bern, Sidlerstrasse 5, 3012 Bern, Switzerland
}

Received 30 January 2001 / Accepted 31 July 2002

\begin{abstract}
We present a statistical study of the post-formation migration of giant planets in a range of initial disk conditions. For given initial conditions we model the evolution of giant planet orbits under the influence of disk, stellar, and mass loss torques. We determine the mass and semi-major axis distribution of surviving planets after disk dissipation, for various disk masses, lifetimes, viscosities, and initial planet masses. The majority of planets migrate too fast and are destroyed via mass transfer onto the central star. Most surviving planets have relatively large orbital semi-major axes of several AU or larger. We conclude that the extrasolar planets observed to date, particularly those with small semi-major axes, represent only a small fraction $(\sim 25 \%$ to $33 \%$ ) of a larger cohort of giant planets around solar-type stars, and many undetected giant planets must exist at large ( $>1-2$ AU) distances from their parent stars. As sensitivity and completion of the observed sample increase with time, this distant majority population of giant planets should be revealed. We find that the current distribution of extrasolar giant planet masses implies that high mass (more than 1-2 Jupiter masses) giant planet formation must be relatively rare. Finally, our simulations imply that the efficiency of giant planet formation must be high: at least $10 \%$ and perhaps as many as $80 \%$ of solar-type stars possess giant planets during their pre-main sequence phase. These predictions, including those for pre-main sequence stars, are testable with the next generation of ground- and space-based planet detection techniques.
\end{abstract}

Key words. solar system: formation - stars: circumstellar matter - stars: planetary systems - stars: statistics

\section{Introduction}

Extrasolar giant planets (EGPs) have been detected by the radial velocity method at orbital distances from several AUs (e.g., 47 UMa c, Gl614b, $\epsilon$ Eri b, 55 Cnc d) to several hundredths of an AU (51 Peg b et al.) from their central stars (see Mayor \& Queloz 1995; Butler \& Marcy 1996; Butler et al. 1997; Noyes et al. 1997; Cochran et al. 1997; Santos et al. 2000; Vogt et al. 2000; Hatzes et al. 2000; Butler et al. 2001; Santos et al. 2001; Fischer et al. 2002; Marcy et al. 2002; and many others; see also the review by Marcy et al. 2000a). Unless giant planets form in place within $1 \mathrm{AU}$ of low mass stars - unlikely in the context of published formation models (see, for example, Guillot et al. 1996) - the observed range of orbital semi-major axes implies that dramatic orbital changes occur after formation. Previous work showed that in gaseous disks and even subsequent particulate disks, giant planets can move from formation distances of around $5 \mathrm{AU}$ to a wide range of final distances, a process known as orbital migration (Lin \& Papaloizou 1986; Lin et al. 1996; Takeuchi et al. 1996; Ward 1997a, 1997b; Trilling et al. 1998; Murray et al. 1998; Bryden et al. 1999; Kley 1999, 2000; Del Popolo et al. 2001; Tanaka et al. 2002). The first direct

Send offprint requests to: D. E. Trilling, e-mail: trilling@hep.upenn.edu determination of the radius of an extrasolar giant planet via transit measurement (Charbonneau et al. 2000; Henry et al. 2000) supports rapid inward migration of giant planets, since the large planetary radius requires close proximity to the parent star when the planet's internal entropy was much larger than at present (Guillot et al. 1996; Burrows et al. 2000), and formation in place is generally considered implausible (see, e.g., Guillot et al. 1996). Early inward migration is commensurate with the idea of migration caused by disk-planet interactions, as disk lifetimes are not longer than $10^{7}$ years (e.g., Zuckerman et al. 1995).

In this work, we address the following questions: (1) How efficient is orbital migration? (2) What population of planets survives the migration process? and (3) How does this produced population compare to the observed EGP population? We answer these questions by allowing a large population of giant planets to evolve and migrate in circumstellar disks with various initial conditions (one planet per disk) and determining the final semi-major axis and mass distributions. We do not employ any stopping mechanisms, but instead allow only those planets to survive whose migration timescales are longer than their disks' lifetimes. We compare the final semi-major axis and mass distributions of the surviving model planets to those of the observed EGPs. We reproduce the fraction of planets 
in small orbits, and predict the frequency of and orbital distributions for giant planets that are as yet unobservable. Of those stars which do form giant planets, a few percent should have giant planets ultimately residing in small orbits, and around one quarter of stars which form planets retain planets in orbits of semi-major axis several AU and beyond. Given the current discovery statistics and their uncertainties, our results imply that giant planet formation is very efficient around low-mass stars: we find that $10 \%$ to $80 \%$ of young stars form planets, with the uncertainty dominated by the initial planetary mass distribution (and with some poorly-known uncertainties associated with discovery statistics). The presently detectable portion of the giant planet population represents only about $25 \%$ to $33 \%$ of the total extant population of giant planets.

The model we present here is a simple one with a minimum set of physical assumptions. By neglecting specific stopping mechanisms, we provide results that represent a baseline for planet formation statistics, bereft of specific additional assumptions that any given stopping mechanism would require. It is hoped that the calculations and results presented here are transparent enough that others can use them to explore the particular effects that specific assumed stopping mechanisms would have. In short, we use the simplest possible model in order to explore the consequences of and for planet formation.

\section{Assumptions in the model}

In order to approach these problems in a simple and clear way, we must make several assumptions about the problem of planetary migration. These assumptions are listed here and explained below: (1) we use the simple impulse approximation for the type II migration; (2) we employ no ad hoc mechanisms to stop planetary migration; (3) we assume that gas accretion onto planets migrating in a gap is small, and neglect this accretion; and (4) we neglect type I migration.

\subsection{Impulse approximation}

In the migration model presented here, we use the impulse approximation described in Lin \& Papaloizou (1986) to calculate the torques between the planet and disk and determine the planet's orbital motion. We have previously shown (Trilling et al. 1998) that the impulse approximation yields quantitatively similar results to much more computationally intensive methods of calculating orbital migration (for example, the WKB approximation of Takeuchi et al. 1996) in the dissipation regime relevant to this work. Given our current knowledge of the migration process, we prefer to explore the consequences of the simplest possible model rather than to add complexity which does not guarantee more accurate results.

\subsection{Stopping mechanisms}

Halting a giant planet's inward migration at small semi-major axes is even less well understood than initiating and maintaining migration. Various stopping mechanisms have been proposed, including tides (Lin et al. 1996; Trilling et al. 1998); magnetic cavities (Lin et al. 1996); scattering by multiple planetesimals (Weidenschilling \& Marzari 1996); resonant interactions by two (or more) protoplanets (Kley 2000); and mass transfer from the planet onto the star (Trilling et al. 1998). All stopping mechanisms have problems: Tides are insufficient because the magnitude of the tidal torque is hardly ever enough to counterbalance the inward torque; magnetic cavities have not been demonstrated to work in a quantitative way; the presence of more than one planetesimal or planet requires a great deal of serendipity; and mass transfer only works in a small percentage of cases. Additionally, stopping mechanisms must not only produce 51 Peg b-like planets, at small orbital distances, but must also produce planets like HD $108147 \mathrm{~b}$ at $0.1 \mathrm{AU}, \mathrm{HD} 28185 \mathrm{~b}$ at 1.0 AU, and 47 UMa c at 3.8 AU (Pepe et al. 2002; Santos et al. 2001; Fischer et al. 2002). None of the above mechanisms adequately halts planets at the wide range of observed distances from the parent stars.

In this work, we presume that none of the above mechanisms are effective in stopping more than a small fraction of migrating planets, and instead consider planets stopping for what is essentially a statistical reason: the planet stops migrating when the disk dissipates. We show that by considering a physically reasonable distribution of initial conditions for planets and disks, our model produces a range of final orbital semi-major axes for extrasolar planets - including small, 51 Peg b-like separations - without resorting to any exotic stopping mechanisms. It is not necessary to produce many planets very close to their stars: the current detection rate suggests that approximately $1 \%$ of low-mass main sequence stars (F, G, K dwarfs) have extrasolar giant planets in small orbits (Vogt et al. 2000), and extrasolar planets in all orbits exist around perhaps a few percent of all stars in the radial velocity surveys (see, e.g., Marcy et al. 2000a). We note in passing that one M-dwarf exhibits two companion objects of at least 0.5 and 2 Jupiter masses (Marcy et al. 1998; Delfosse et al. 1998; Marcy et al. 2001a); the statistics for M dwarfs are thus very poor and indeed the environment for planet formation may be very different from that of the more massive dwarfs. In what follows we confine ourselves to $\mathrm{F}$ through $\mathrm{K}$ stars and refer to these conveniently as low-mass stars.

\subsection{Planetary mass accretion during migration}

Giant planets form gaps in their gaseous circumstellar disks; when this occurs, $\dot{M}_{\text {p }}$, the planetary mass accretion rate, drops substantially from its pre-gap value as the local surface density is substantially reduced. It is an outstanding question how much, if any, material may flow across the gap from the disk and be accreted onto the planet (see Bryden et al. 1999; Kley 1999; Bryden et al. 2000; Kley 2000; Lin et al. 2000; Kley et al. 2001). The results shown in those works suggest that accretion timescales $\left(\tau_{\text {acc }} \sim M_{\mathrm{p}} / \dot{M}_{\mathrm{p}}\right)$ are between $3 \times 10^{5}$ and $3 \times 10^{6}$ years for the viscosities, mass ratios, and other parameters we use in our models. We have adopted a characteristic accretion timescale of $10^{6}$ years and performed several tests to determine the effect of including mass accretion in the migration models (Fig. 1). Note that the accretion timescale is 


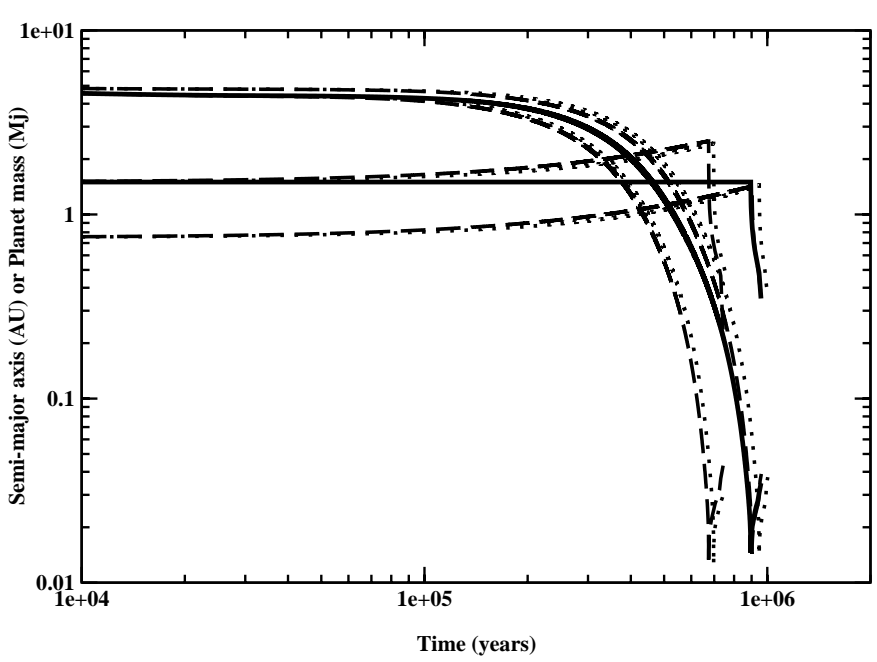

Fig. 1. Planetary semi-major axis (in $\mathrm{AU}$ ) and planet mass (in $M_{\mathrm{J}}$ ) versus time for migrating planets. Shown here are migrating planets' semi-major axes (generally decreasing curves beginning at 5.2 AU) and masses (generally flat or slightly increasing curves beginning near $1 M_{\mathrm{J}}$ ) for the following five cases: the baseline case, with no mass accretion during migration (solid black line); two cases with linear mass accretion during migration (dashed lines); and two cases with exponential mass accretion during migration (dotted lines). For the no-accretion case, the initial planet mass is $1.5 M_{\mathrm{J}}$. For the accretion cases, the initial planet masses are $0.75 M_{\mathrm{J}}$ and $1.5 M_{\mathrm{J}}$; the growth timescale is $10^{6}$ years. The set of two semi-major axis curves above and to the right of the baseline case represent the two cases with planets initially $0.75 M_{\mathrm{J}}$; the two semi-major axis curves below and to the left of the baseline curve are planets with initial mass $1.5 M_{\mathrm{J}}$. All planets lose mass through Roche lobe overflow when their planetary radii are larger than the Roche radii. This mass loss can be seen by a downturn in planet mass near the end of each run; mass loss produces outward motion to conserve angular momentum (thus the very late outward motion of mass-loss planets). Model runs were halted when planet mass became less than $0.4 M_{\mathrm{J}}$. This figure demonstrates that even allowing for mass accretion during migration, the overall migration timescale is not very different from that of the baseline (no accretion) case. Because of this, for simplicity, we neglect mass accretion during migration for the models in the statistical study.

comparable to the migration timescale; we allow both linear and exponential growth with this characteristic timescale. The results from these test cases show that when all disk parameters are held constant, the range of variation between the migration timescales without mass accretion and timescales with mass accretion (all cases, linear and exponential) is around 25\% from the no-growth migration timescale of $\sim 10^{6}$ years (Fig. 1).

Including mass accretion for a population of migrating planets has a small effect on the overall results, at best. For more massive planets, which migrate quite slowly anyway (see Sects. 3.2 and 4), the effect of considering mass accretion is very much smaller, or completely negligible. Additionally, the primary parameters which drive migration (diskmass, viscosity, disk lifetime) are not known to better than $25 \%$, and even the mass accretion rate itself is a product of some underlying assumptions about the flow of material near, around, and onto a migrating planet. Attention to the detail of mass accretion during migration is not warranted in the face of these larger unknowns; instead, we choose to use a simpler model (ignoring post-gap formation accretion) which may neglect some details for the sake of clarity and understanding. Lastly, because migration timescales are only affected by mass accretion in a minor way, the overall semi-major axis and final mass distributions of the statistical study (see below) do not change significantly in the presence or absence of mass accretion. We therefore neglect mass accretion for the remainder of the present work; all subsequent discussions concerning the statistical study implicitly refer to model runs without mass accretion.

\subsection{Type I migration}

Giant planets, because of their large ratio of hydrogen and helium relative to heavier elements, must form in gaseous protoplanetary disks. Regardless of whether a giant planet forms by core accretion (e.g., Wuchterl et al. 2000) or by direct collapse (e.g., Boss 1997, 1998, 2000, 2001), when a planet grows to a certain mass $\left(\sim 10-30 M_{\text {Earth }}\right.$, although there is some debate about what this critical mass actually is), a gap is formed in the disk and spiral density waves travel away from the planet (see, for example, Goldreich \& Tremaine 1980; Lin \& Papaloizou 1986; Ward \& Hourigan 1989; Lin \& Papaloizou 1993). Interactions of these waves at the planet's Lindblad resonances transfer angular momentum between the planet and the disk; this angular momentum exchange in turn produces a change in orbital distance of the planet. Usually (but not always), these interactions lead to a net loss of angular momentum and a decrease in the planet's orbital semi-major axis. Such planet-disk interactions have been described in detail (see, for example, Goldreich \& Tremaine 1980; Ward \& Hourigan 1989; Lin \& Papaloizou 1986, 1993; Lin et al. 2000; Ward \& Hahn 2000). The type of migration executed with a planet in a gap is referred to as type II (see Ward \& Hahn 2000).

So-called type I migration is executed by bodies which are not massive enough to form gaps in their disks (i.e., less than 10-30 $M_{\text {Earth }}$ ) (Ward 1997a, 1997b). Ward has shown that type I migration may be very fast (less than $10^{6}$ or even $10^{5}$ years) which is obviously detrimental to planet growth and survival: if migration timescales are so short for Earth mass objects, it is unclear how any planet could survive long enough to accrete enough gas mass to open a gap and initiate the slower type II migration (which itself is perilous for planet survival). Clearly, there is a problem with planet survival in the face of type I migration. We do not intend to solve this problem in this paper. Instead, we neglect type I migration for the following reasons.

If type I migration were really as dangerously efficient as has been proposed, then formation and survival of planets (particular at distances greater than $0.1 \mathrm{AU}$ ) would be nearly impossible, since the type I migration timescales are shorter than any possible disk lifetime. But, since we know that planets survived both at small and intermediate semi-major axes (many EGPs) and also relatively large semi-major axes (Jupiter), type I migration cannot be as universally destructive as proposed. For lack of a better quantification of the type I effect, in the present 
work we neglect the effect of type I migration on the initial conditions used in our models. Furthermore, all planets we consider in this paper are too massive to undergo type I migration. Thus, we can safely neglect type I effects in our calculations. We reiterate that this does statement does not refute type I migration nor the importance of understanding and characterizing its timescales; however, it is clear that for planets which have reached $\geq 1$ Jupiter mass via any formation mechanism, type I migration is no longer relevant.

\section{Planet migration and the statistical study of planet survival}

\subsection{The migration model and disk properties}

We have applied a model of disk-induced migration (Trilling et al. 1998) to a set of planetary systems whose initial characteristics are distributed over a representative range of planet formation environments. We use the same method and model as used in Trilling et al. (1998), which includes torques on the planet due to disk-planet interactions, due to star-planet tidal torques, and due to planetary mass loss. Mass loss significantly prolongs planetary lifetimes for only a very small population of model planets, but is included here for completeness. Starplanet tidal torques affect a larger population, but we shall see that, by far, the largest effect is the magnitude of the disk-planet torque. A planet's survival, which is enabled essentially entirely by dissipation of the gaseous disk before infall onto the star, depends on its mass, and on the disk mass, disk lifetime, and disk viscosity.

We have performed a statistical study of planet fates in systems in which each of disk mass, disk lifetime, and disk viscosity is varied in turn. Initial planet masses are taken to be integral values between 1 and 5 Jupiter masses (1 Jupiter mass $=1 M_{\mathrm{J}}=2 \times 10^{30} \mathrm{~g}$ ), inclusive, evenly distributed, with one planet per system. Planetary accretion after gap formation is neglected (see Sect. 2.3). For the three varied parameters, we adopt nominal values and vary each parameter over a range spanning a factor of 30 larger and smaller than the nominal value, with 200 model runs evenly spaced per decade in $\log$ space, for a total of 600 model runs per planet per varied parameter. For example, in varying disk mass, we adopt a nominal disk mass of $0.02 M_{\odot}$ (after Beckwith et al. 2000), and calculate model cases between $0.02 / 30$ and $0.02 \times 30 M_{\odot}$, or 0.0007 and $0.6 M_{\odot}$. This range spans disk masses from less than the minimum mass disk for a system containing a Jupiter to a disk so massive that direct binary star production is more likely than giant planet formation (see, for example, reviews by Beckwith \& Sargent 1993 and Beckwith et al. 2000). Over this entire range we test 600 model runs in evenly spaced intervals of $0.005 \log$ units. Similarly, we use a nominal disk lifetime and alpha viscosity of $3 \times 10^{6}$ years and $3 \times 10^{-3}$, respectively (after Zuckerman et al. 1995; Shakura \& Sunyaev 1973; and Trilling et al. 1998) and test parameter values from disk lifetimes of $10^{5}$ to $9 \times 10^{7}$ years and disk viscosities of $10^{-4}$ to $9 \times 10^{-2}$. In all, we carried out 1800 model runs per planet mass and 9000 model runs total; each model run has one parameter varied while the other two are given their nominal values. This very fine sampling of disk parameters allows us to simulate a continuum of physical properties of planet-forming systems. All planets' initial orbital semi-major axes are 5.2 AU. Giant planets forming farther out would spend more time migrating inward, all else being equal, and hence would have a larger chance of survival (see below).

The result of our computations is a three-dimensional grid of models, based on the nominal values and spanning the range of parameter space, for each initial planet mass. To produce a Gaussian statistical population in which the nominal value is most likely and the extreme values are least likely, the significance of each model run was weighted by the Gaussian probability of the value of the varied parameter according to the standard Gaussian formalism:

$P(u)=\frac{1}{\sigma \sqrt{2 \pi}} \mathrm{e}^{-\left\{\frac{1}{2}\left(\frac{u-u_{0}}{\sigma}\right)^{2}\right\}}$

where $u=\log x$ and $u_{0}=\log x_{0}, x$ is equal to the value of the varied parameter, $x_{0}$ is the nominal value for the parameter being varied, and where $\sigma$, which describes the width in $\log$ space of the Gaussian parameter distribution we use, is equal to 0.5 . In other words, the half-width of the Gaussian physical parameter distribution is a factor of $\sqrt{10}$, or around 3.16; thus, a parameter value which is 3.16 times greater or less than the nominal value has a probability one half of the probability that of the nominal value: $P\left(u_{\mathrm{h}}\right)=\frac{1}{2} P\left(u_{0}\right)$ for $u_{\mathrm{h}}=u_{0} \pm 0.5$ which is the same as $x_{\mathrm{h}}=x_{0}(3.16)^{ \pm 1}$.

We use this Gaussian weighting in all results discussed in this paper. Weighting is achieved by assigning a significance to each surviving model planet as $\delta \times P(u)$ where $\delta$ is equal to 1 for planets which survive and 0 for planets which do not. Thus, in production of histograms and discussing the ensemble results for the 9000 model runs, we explicitly incorporate the probability of the planet's formation conditions and circumstellar disk. Thus, surviving planets from extreme disks whose initial conditions are extremely unlikely are not overrepresented in the statistical results. Note that because probabilities are assigned after all model runs are completed and are simple functions of the nominal values and a description of a Gaussian distribution, other probability weightings can be installed to represent other experiments. Future work includes deriving both $x_{0}$ and $\sigma$ more directly from observations (see, e.g., Gullbring et al. 1998; Hartmann et al. 1998) and testing non-Gaussian probability distributions.

Our models calculate orbital evolution for $10^{10}$ model years, and planets are defined to have survived when they have non-zero semi-major axes after this time. Very few planets (much less than 1\%) undergo significant orbital evolution (due to star-planet tides) after the disk dissipates.

\subsection{Results of the statistical study}

The results from our statistical study are shown in Figs. 2 and 3. Of the 9000 initial planets, $30 \%$ survive. This survival rate assumes a flat mass function (see below), and is normalized to the total probability of all surviving planets; in this and subsequent discussions, all results are post-weighting. We find that around $0.8 \%$ of the initial planets have final semi-major axes less than 


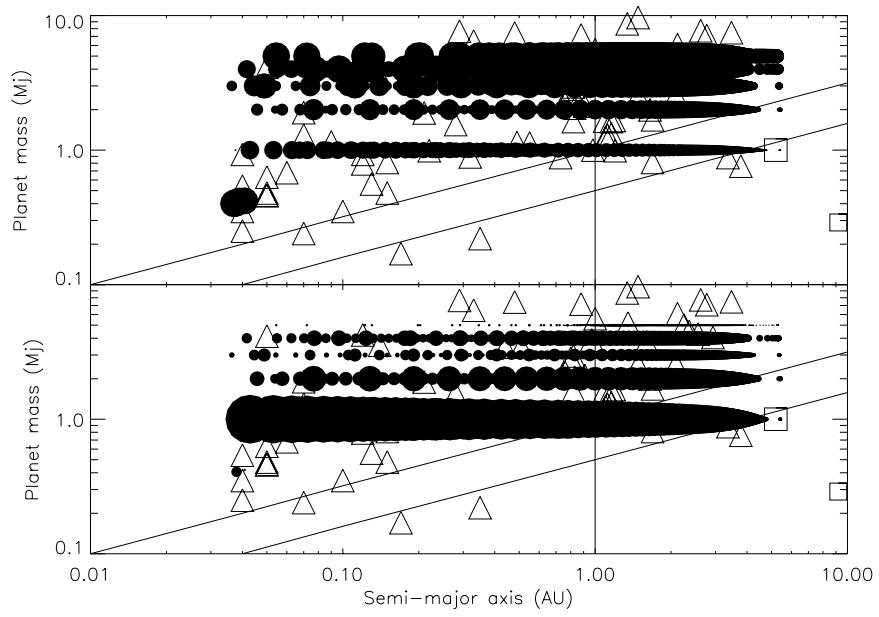

Fig. 2. Planet mass versus heliocentric distance for observed (triangles) and model (filled circles) EGPs. $M$ sin $i$ (minimum planet mass) is used for all observed EGPs. The open squares are Jupiter and Saturn. Also shown are the 5- and 10-sigma detection limits from the radial velocity method of detecting extrasolar planets (lower and upper diagonal lines). These limits assume an rms precision of $3 \mathrm{~m} / \mathrm{s}$, which is typical for the very high precision radial velocity surveys (Butler et al. 1996). The vertical solid line at $1 \mathrm{AU}$ corresponds to the completion limit of the radial velocity observations (G. Marcy, pers. comm.). The two panels show results for the flat (upper) and preferred (lower) mass function (see text). The radius of the point for each model planet represents that model's probability (the same arbitrary relative scaling is used in both panels). Beyond $1 \mathrm{AU}$, some EGPs have been detected, but many others may also exist, undetected to date. Most observed EGPs are found at small semi-major axes because these planets are easiest to detect. The majority of model EGPs which survive the formation and migration processes have large semi-major axes (see text). These two statements imply that a large population of EGPs exists that has not yet been detected, a prediction made by this work. Observed EGP data is as of January, 2002. Model masses are integral masses $1 M_{\mathrm{J}} \leq M_{\mathrm{p}} \leq 5 M_{\mathrm{J}}$.

0.1 AU. Approximately $6.5 \%$ have final semi-major axes between 0.1 and $1 \mathrm{AU}$. The rest of the surviving planets (23\% of the original planets) have final distances from their stars greater than $1 \mathrm{AU}$. We find that $2.5 \%$ of all surviving planets are found at less than $0.1 \mathrm{AU} ; 21 \%$ are found between 0.1 and $1 \mathrm{AU}$; and $76 \%$ of surviving planets have semi-major axes greater than $1 \mathrm{AU}$. The majority $(70 \%)$ of the population of initial planets migrates too fast; these planets lose their mass onto the central star (by Roche lobe overflow), and do not survive. As noted in Sect. 2.2, other stopping mechanisms at the inner edge of the disk may operate and would increase somewhat the fraction of planets retained in orbits with very small semi-major axes. These mechanisms will have a small or negligible effect on the overall statistics, except for the possible existence of a magnetic cavity, which effect on the migration has yet to be quantified.

The most likely fate of a planet which forms near 5 AU is that it migrates inward too fast and ultimately is lost onto the central star. The vast majority of planets that do survive, though, tend to reside at greater than $1 \mathrm{AU}$ from their central stars. Our statistical study is robust because we have sampled planet migration over a broad distribution of disk masses,

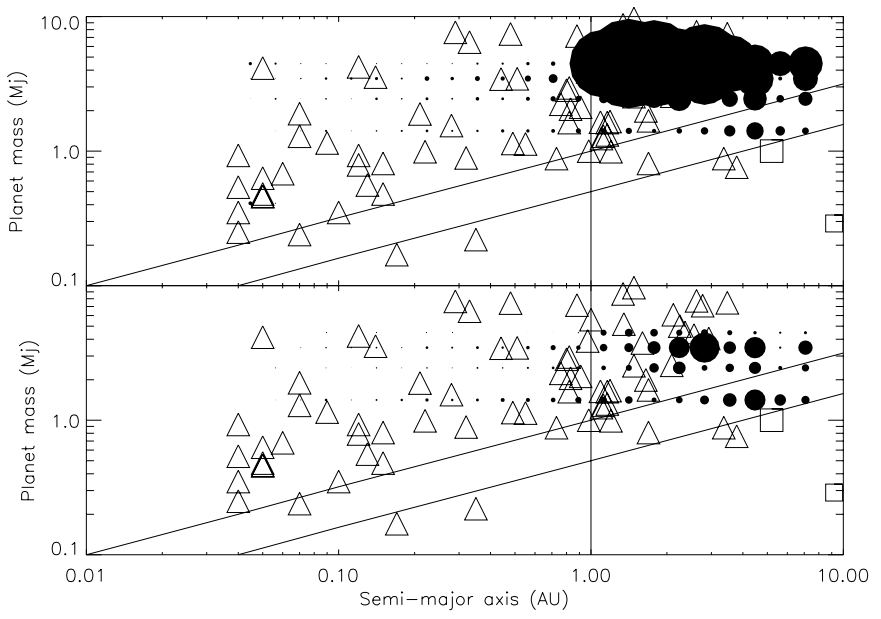

Fig. 3. Same as in Fig. 2 but for the total overall probability of planet survival in given semi-major axis and mass bins; both semi-major axis and mass bins are $0.1 \log$ units wide, centered on $0.05 \log$ units. The radii of the filled circles again show the probability of model planets populating that bin, but a different (arbitrary) probability normalization is used than for Fig. 2 to show circle size more clearly; the same probability normalization is used for both panels here. As before, the upper panel is shows results for model planets with a flat initial mass distribution; the lower panel is for results with the preferred initial mass distribution.

viscosities, lifetimes and initial planet masses: the variables that are key to migration and its termination. We have also carried out an initial study of the effects of initial semi-major axis on planet migration and survival. While it is not easy or obvious to parameterize these results, it is clear that for identical initial disk conditions, planets which form farther out migrate less rapidly. This is because the surface density is lower at larger semi-major axes, so that the torque between the planet and disk is less. The effect on planet survival is that more planets, of lower masses, survive (see below). Therefore, if $5 \mathrm{AU}$ is the minimum semi-major axis for giant planet formation, then the results presented here represent a minimum for planet survival; conversely, if $5 \mathrm{AU}$ corresponds to the largest semi-major axis for planet formation, then the planet survival found here is the upper limit. We choose 5.2 AU as a nominal middle ground. The fraction of planets which survive, the ratio of planets at small semi-major axes compared to at large semi-major axes, and the overall shape of the surviving planet mass distribution are quite similar even when somewhat different initial semimajor axes are considered (see below).

\section{Final orbital semi-major axis distribution}

We have analyzed our statistical results in terms of the final distribution of orbital semi-major axes of the planets, in comparison to the observed EGP distribution. The results are shown in Fig. 4. The observed EGP distribution is relatively flat, that is, equal numbers of planets throughout the range of 0.05 to around $0.7 \mathrm{AU}$; there is the suggestion of a rise from $0.7 \mathrm{AU}$ through just over $1 \mathrm{AU}$; and a decrease at greater semi-major axes than just over $1 \mathrm{AU}$ (this decrease is due at least in part to observational bias). (The exception to this apparently flat 
distribution is the slight enhancement in planets at orbital distances around 0.05 AU. In our previous work (Trilling et al. 1998), we described how tidal and mass loss torques can cause a small "piling-up" of planets near the tidal limit, which is typically around $0.05 \mathrm{AU}$.) In contrast, the surviving planets in our statistical study are predominantly at large ( $>1 \mathrm{AU})$ semi-major axes, including a rise at just under $1 \mathrm{AU}$ to a peak at 2-4 AU. The hint of an upturn in the observed distribution at less than 1 AU echoes the pattern in the model distribution and may suggest the larger population that we predict at greater than $1 \mathrm{AU}$. The physical explanation for the peak in model planets at separations greater than $1 \mathrm{AU}$ is that the time during which a planet is migrating ( $10^{5}$ to $10^{6}$ years) can be relatively short compared to both the lifetime of the disk $\left(\sim 10^{7}\right.$ years $)$ and the timescale required to initiate migration (variable, but at least $10^{6}$ years, or more) (Trilling et al. 1998). Therefore, the number of planets which survive at small distances $(<1 \mathrm{AU})$ is small. The majority of planets that survive do so by creating very large gaps in their disks and by requiring long timescales to excavate the farthest regions of their large gaps, as follows.

More massive planets create larger gaps than smaller planets, everything else being equal (Takeuchi et al. 1996; Trilling et al. 1998). For the most massive planets, the length of time it takes to fully form the gap can be quite long, approaching or even surpassing the lifetime of the disk, as such gaps are many AUs wide. The gap is completely formed when it reaches its equilibrium size (see Takeuchi et al. 1996 and Trilling et al. 1998). During the stage in which a gap is growing but has not yet reached its full equilibrium size, there is no type I migration since the planet has already opened a gap; there is also little type II migration because most Lindblad resonances already fall within the growing gap. Thus, the planet is hardly migrating during this time in which the gap is still growing to its equilibrium size. If the timescale for gap growth is longer than the disk lifetime then the planet will not migrate far. Therefore we find that, overall, close-in surviving model planets have smaller masses and distant surviving models planets have larger masses; this rough trend is seen in the observed data as well (see below). Note that relatively large mass planets (compared to the disk mass) are required, in general, for these arguments about large gap formation to be applicable.

Since the vast majority of planets either migrate too fast and are lost onto the star or remain out near their formation location, disk and planet parameters must be "just right" to halt a planet at small distances $(<1 \mathrm{AU})$. The conclusion of this analysis is that it is very difficult to make planets survive at small distances from the parent star, and that to do so requires a large population of giant planets which still reside at large distances from their parent star as well as a population which has migrated too fast and been lost onto the central star. From our model results, we find that for every planet found at small semi-major axes, around 3 planets must exist at much larger distances, depending on the initial mass distribution (see below). The radial velocity technique is most sensitive to planets at small semi-major axes, because the magnitude of the stellar wobble is greatest for close-in planets. Additionally, since the highest precision radial velocity surveys have relatively short baselines of data, planets with longer periods are only

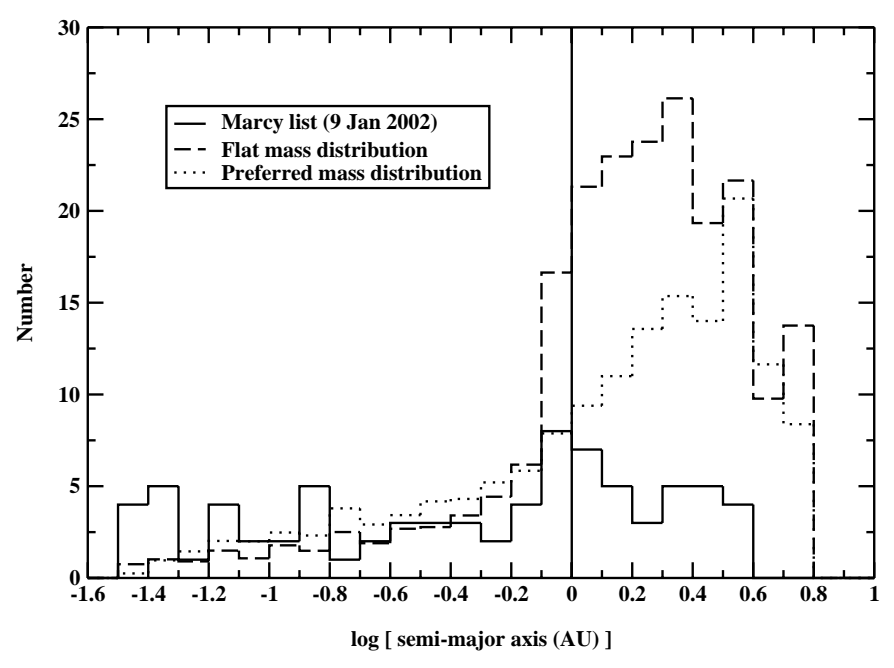

Fig. 4. Histogram showing orbital semi-major axes for observed (solid line) and model (dashed and dotted) EGPs. Each model histogram is normalized such that the number of model planets with semi-major axes less than $1 \mathrm{AU}$ is equal to the number of observed EGPs with $a<1 \mathrm{AU}$. The vertical line corresponds to $1 \mathrm{AU}$, the completion limit for radial velocity surveys. Most model EGPs are to the right of the vertical line, i.e., at larger semi-major axes than would have been detected thus far by the radial velocity searches. The dashed line is for a flat mass function; the dotted line is for the preferred mass function (see text). Bins are 0.1 units in the log of semi-major axis.

beginning to be identified (e.g., $55 \mathrm{Cnc}$ d, with $\sim 15$ year orbit, Marcy et al. 2002). Therefore, the majority of the extant population of giant planets we predict would, at present, be beyond the detection capabilities of the radial velocity searches. In other words, a large population of Jupiter-like planets is as yet undetected.

\section{Implications}

\subsection{Giant planet mass distribution}

We have also analyzed our surviving statistical population for its distribution of final planet mass, in comparison to the observed EGP mass distribution. This comparison is complicated by the fact that for most EGPs, the known quantity is not planetary mass but $M_{\mathrm{p}} \sin i$. We assume $\sin i=1$, for simplicity. We shall see in any case that it is not the absolute mass that matters but the slope and shape of the mass distribution curve. The slope and shape will be roughly unchanged between $M_{\mathrm{p}} \sin i$ and $M_{\mathrm{p}}$ assuming a random distribution of system inclinations relative to the Earth. For a random distribution of inclinations, the average of $\sin i$ is $\pi / 4$. With increasing numbers of extrasolar planets known, the average $\sin i$ for the population gets closer to the value for a random distribution. However, because of the detection bias of the radial velocity technique (biased towards detection of systems with $i$ near 90 degrees), the average of $\sin i$ for the detected systems is actually between $\pi / 4$ and unity (Marcy et al. 2000a).

The comparison among mass functions used in this work is shown in Fig. 5. By assumption, in our model we have started with equal numbers of planets with masses $1,2,3,4$, and $5 M_{\mathrm{J}}$ (i.e., after accretion has taken place), or a flat initial mass 


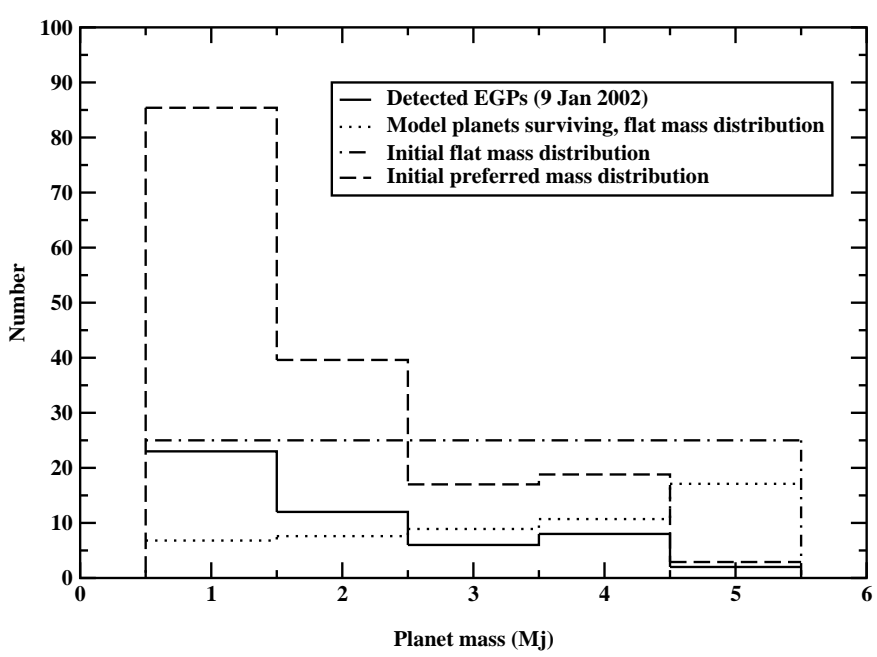

Fig. 5. Histogram showing final planet mass distribution for observed EGPs (solid line). Also shown are the flat model initial mass distribution (dot-dash line), the surviving population of model planets produced from this flat mass function (dotted line), and the preferred initial mass distribution (dashed line) required to produce the observed mass function (see text). For the observed EGPs, $M_{\mathrm{p}} \sin i$ is used. The preferred mass function is described in the text. Mass bins are $1 M_{\mathrm{J}}$ wide, centered on integral masses. The model mass distributions are normalized to the number of observed EGPs (solid line).

distribution. As shown in Figs. 2, 3, and 5, most of the surviving planets in the statistical study are the more massive planets; planets with the largest initial mass $\left(5 M_{\mathrm{J}}\right)$ survive preferentially. The observed EGP mass distribution, however, is biased toward smaller mass planets (Fig. 5). Since the radial velocity technique is most sensitive to larger masses, the relative dearth of large mass planets discovered is significant. The present observed EGP population can instead be obtained with an initial mass distribution biased strongly toward smaller mass giant planets. This "preferred" initial distribution is shown in Fig. 5 as the dashed line. The preferred mass function is derived from the observed mass distribution and the ratio of initial model planets to surviving model planets:

\# of initial preferred mass distribution planets with $M_{\mathrm{i}}$ \# of observed EGPs with $M_{\mathrm{i}}$

\# of initial flat mass distribution planets with $M_{\mathrm{i}}$

\# of surviving flat mass distribution planets with $M_{\mathrm{i}}$

where initial planet masses are $M_{\mathrm{i}}=1,2,3,4$, and $5 M_{\mathrm{J}}$ and mass bins of $\pm 0.5 M_{\mathrm{J}}$ are used. The preferred initial mass distribution by definition produces a final mass distribution identical to that which is presently observed.

Model planet masses and semi-major axes produced using the preferred the initial mass distribution are shown in Figs. 2 and 3. The final distributions of semi-major axis and planet mass produced using the preferred initial mass distribution are shown in Figs. 4 and 5. As with a flat initial mass distribution, in order to produce the observed extrasolar planet characteristics from the preferred initial mass distribution, many planets with large semi-major axes must be produced. The preferred initial mass distribution results in $0.2 \%$ of all initial planets surviving with separations less than $0.1 \mathrm{AU} ; 1.6 \%$ of all initial planets survive and have $0.1 \mathrm{AU}<a<1 \mathrm{AU}$; and $3.8 \%$ of all initial planets survive and have $a>1$ AU. These percentages correspond to $4.4 \%, 28 \%$, and $68 \%$ of all surviving planets to be found in those three semi-major axis ranges, respectively. Thus, the ratio of as-yet undetected planets to detected planets should be around 2:1, compared to a ratio of $3: 1$ obtained with the flat mass distribution. Thus, the total percentage of stars with planets must be 3-4 times greater than the percentage given by the current observational results. With the preferred initial mass distribution, $5.6 \%$ of all initial planets survive at any semi-major axis; with the flat initial mass distribution, this total survival rate is $31 \%$. With the preferred initial mass distribution, fewer planets survive than in the case of the flat initial mass distribution because the smaller mass planets that dominate the preferred initial distribution preferentially are destroyed ${ }^{1}$. Our results imply that there must still be a substantial population of giant planets at large semi-major axis that has not yet been detected. The ratio of extant planets at large semi-major axes to extant planets at small semi-major axes is less when the preferred initial mass distribution is used (around 2 to 1) compared to when the nominal (flat) initial mass distribution is used (closer to 3 to 1 ).

\subsection{Planet-forming efficiency}

Planet forming efficiency, that is, the number of stars that form giant planets, must be relatively high. This is because many planets which form are subsequently destroyed, and many planets which exist have not yet been detected, as follows:

$$
\begin{aligned}
& \frac{1 \text { planet detected }}{100 \text { stars }} \times \frac{3 \text { to } 4 \text { extant planets }}{1 \text { planet detected }} \\
& \times \frac{20 \text { planets formed }}{1 \text { to } 6 \text { extant planets }} \sim \frac{10-80 \text { planets formed }}{100 \text { stars }} .
\end{aligned}
$$

The first term, the number of observed stars which have closein planets, is from Vogt et al. (2000) and Marcy et al. (2000a); the second term, that 2 to 3 planets exist for each planet which has been discovered so far, is from Sects. 4 and 5.1; the third term, the fraction of planets formed which survive, is from Sects. 3.2 and 5.1. Note that our model only incorporates 1 planet per star, so this means $10 \%$ to $80 \%$ of $\mathrm{F}, \mathrm{G}$, and $\mathrm{K}$ dwarf stars form (at least) one giant planet. This is a very large percentage, which bodes well for observing large numbers of giant planets around young stars (see below).

\footnotetext{
1 Ward (1997a, 1997b) concludes that type II migration timescales are independent of planet mass. This conclusion is valid in the limit that the planet's mass is small compared to the disk mass, resulting in a planet which is fully entrained in the viscous evolution of the disk. In the case of a finite mass ratio between planet and disk, however, there is a feedback torque from the planet onto the disk, and the planetary migration timescale is seen to be a function of planetary masses (Lin \& Papaloizou 1986; Trilling et al. 1998; Bryden et al. 2000; Nelson et al. 2000; Kley 2000; D'Angelo et al. 2002). In the regime in which we are working, planetary migration timescales are functions of planet masses in the sense that, in general, smaller planets migrate faster and more massive planets migrate more slowly. This is partially a function of gap size, as described in Sect. 4.
} 
The primary source of uncertainty is the third term, based on the unknown initial mass distribution.

Our model suggests a high efficiency of planet formation. Since planets more easily survive migration in lower mass disks, planet formation in such disks leads to greater planet survival. Additionally, the shape of the preferred initial mass distribution suggests a natural cutoff towards larger mass planets: larger mass planets may be more difficult to form (in agreement with Lineweaver \& Grether 2002). In other words, giant planet formation must be a relatively efficient use of the disk gas: it cannot be a process requiring primarily disks at the upper end of the mass range we have considered, but must also occur in disks with masses closer to the minimum we have selected. Our minimum mass disk is slightly less than the mass of Jupiter itself, and represents an extremum. Exclusion of extreme diskmasses from the cohort changes the overall required efficiency very little because these extreme cases have very low probabilities and therefore do not contribute much to the total survival probability. Overall we require that the smallor moderate-disk masses participate in the formation of giant planets, and these giant planets are then overrepresented in the final distribution because of slower migration rates.

Finally, it is important to emphasize that high planet forming efficiency does not mean that most low mass stars currently have Jupiter-like planets. Planetary migration is very unforgiving: our statistical study shows that around $70 \%$ to almost $95 \%$ of formed planets migrate too fast and meet their demise at their central stars. A large percentage (10\% to $80 \%)$ of stars and disks must produce planets so that a few percent may survive close to their central stars and be detected by radial velocity searches. But there should then be a population of giant planets in intermediate (2-4 AU) and larger orbits, around perhaps $2 \%-3 \%$ or more of $\mathrm{F}, \mathrm{G}$, and $\mathrm{K}$ dwarf stars. This fraction agrees with the estimates of Zucker \& Mazeh (2001b). Giant planet formation may be occurring around many young stars today, and searches for indications of planet formation around these stars should have a high success rate.

\section{Discussion and some uncertainties}

Our preferred initial mass distribution and mechanism for producing close-in giant planets requires a high frequency of planet formation in disks, and a bias toward making giant planets with smaller masses (in agreement with Lineweaver \& Grether 2002). The latter conclusion is robust so long as the distribution of observed system inclinations as seen from Earth is roughly random; that is, as long as the measured $M_{\mathrm{p}} \sin i$ yields actual masses on average less than a factor of two larger. To date, five systems with known inclinations (HD 209458, $55 \mathrm{Cnc}$, HD 210277, $\rho \mathrm{CrB}$, and $\epsilon$ Eri) all have $i \gtrsim 30 \mathrm{de}-$ grees and thus have mass factors less than 2 (Charbonneau et al. 2000; Henry et al. 2000; Trilling \& Brown 1998; Trilling et al. 2000; Greaves et al. 1998). Additionally, by tidal arguments, $v$ And, HD 75289, HD 187123, 51 Peg, and HD 217107 are all constrained to have $i \gtrsim 30$ degrees as well, although there are no direct observations as yet to confirm this (Trilling 2000); the Hipparcos data implies an inclination 25 degrees for $v$ And (Mazeh et al. 1999). Additionally, from dynamical and astrometric arguments, the multiple-planet systems G1876, HD 168443, and $v$ And likely have inclinations that are far from face-on (Laughlin \& Chambers 2001; Marcy et al. 2001b; Chiang et al. 2001). Therefore, most systems for which inclinations are known or suspected appear to have mass factors less than around 2. Astrometric studies of EGP systems show a range of results but few certain inclinations, to date (Zucker \& Mazeh 2000; Halbwachs et al. 2000; Gatewood et al. 2001; Han et al. 2001; Pourbaix 2001; Pourbaix \& Arenou 2001; McGrath et al. 2002).

Giant planet formation is as yet not well enough understood to permit prediction of a preferred initial mass distribution as a function of disk mass. Models in which core accretion precedes accumulation of gas require a substantial surface density of solids to permit accretion to occur within the disk lifetime (Lissauer 1987), with the resulting gas accretion occurring fairly rapidly once a threshold mass of solids is accumulated (Wuchterl et al. 2000). There is thus no simple and direct link between the disk gas mass and the final masses of the giant planets formed. The presence of Uranus and Neptune in our own system is suggestive of a process that is sensitive to depletion of the gas either due to small available amounts of gas beyond a certain distance from the primary, or to long core accretion times that stretch beyond the disk lifetime. However, alternative models for the formation of Uranus and Neptune exist that do not tie them directly to conditions in the 20-30 AU region of the gas disk (Thommes et al. 1999).

In sum, we do not as yet have a direct link between the mass distribution of giant planets and the mass of the disk. Thus our preferred initial mass distribution is a general prediction: whatever mechanism forms giant planets tends to prefer the smaller mass objects. This is at least crudely consistent with the inference that the combined giant planet/brown dwarf mass function appears to show a minimum around approximately $10 M_{\mathrm{J}}$ (Marcy \& Butler 2000; Zucker \& Mazeh 2001a), suggestive of a planet-forming process biased to lower masses and a brown dwarf-forming process (direct collapse or disk instability) that is most efficient at much higher masses.

In our study we did not consider minimum masses below $1 M_{\mathrm{J}}$. The lack of data for objects significantly below $M_{\mathrm{p}} \sin i$ of $0.5 M_{\mathrm{J}}$ hampers our ability to extend the calculations, and we must await further and more sensitive data (e.g., SIM, see below) to establish the frequency of lower mass planets. However, the radial velocity surveys have begun to detect planets with masses as small as $0.12 M_{\mathrm{J}}$ (HD 49674b; Butler et al. 2002). This implies that orbital evolution takes place for Saturn-mass and smaller planets. Further observations will show the effects of planet mass on orbital evolution.

Likewise, we have arbitrarily imposed planet formation at 5.2 AU. Formation of giant planets out to double or even quadruple that distance is possible, based on our own solar system. We have performed a small set of model runs for planets with larger initial semi-major axes, out to $10 \mathrm{AU}$. The overall behavior and survival of these migrating planets is not qualitatively different from that of the entire suite of 9000 models. The general effect of larger initial semi-major axes is an increase in the survival rate (because migration times are longer - see Papaloizou \& Larwood 2000). This would produce a 
lower required planet-forming efficiency and an initial mass distribution which is biased toward slightly higher masses relative to the preferred initial mass distribution, since more small mass planets survive. Eventually, however, a distance must be reached in all disks at which accretion times become too long and giant planet formation yields first to ice giants (Uranus and Neptune) and then debris. The current cohort of close-in giant planets does not provide a constraint on the outer orbital radius at which giant planet formation could occur. A more complete sample of observed EGPs at larger semi-major axes is required. It is possible that production of giant planets is actually preferred at the position in the disk where water ice first condenses out, because the surface density of ice coldtrapped there (and hence of total solids) is very high (Stevenson $\&$ Lunine 1988). However, testing this hypothesis must await a more complete sample of planetary systems.

Our model says nothing about the distribution of orbital eccentricities of the known radial velocity companions. Many of the known planets with semi-major axes beyond the tidal circularization radius have significant eccentricities. This suggests a process of orbital evolution commonly associated with the formation and earliest evolution of giant planets, and yet one which failed to act on our own four giant planets. The discussion of whether radial migration in gaseous or particulate disks produces eccentric orbits is an ongoing one (e.g., Ward 1997a, 1997b; Bryden et al. 1999; Kley 2000; Papaloizou et al. 2001; Murray et al. 2002). The presence of high eccentricities suggests that multiple giant planet formation may be the norm, and that interactions among giant planets after disk migration ends pump up the orbital eccentricities. The stochastic nature of such interactions precludes their inclusion in an analysis such as the one we present. Further, until the orbital inclinations can be determined, an important clue to the genesis of the eccentricities is missing. Regardless of the mechanism(s) by which eccentricity is created, except for extreme cases, our results regarding the semi-major axis distribution are hardly affected. Our statistical results regarding planet formation frequency hinge on the frequency of the close-in giant planets versus those farther out. Additional modest semimajor axis evolution associated with eccentricity pumping does not alter our results significantly. Dramatic three-planet interactions that would leave giant planets in vastly altered orbits and greatly affect our statistics are arguably rare. In summary, while we do not calculate eccentricities induced by gas drag in our one-dimensional model, the results based on the statistics of semi-major axis distribution remain robust.

The migration behavior and orbital evolution of planets in a multiple-giant planet system could differ from the results presented here. In these cases, both the gas-planet interactions and the planet-planet interactions could cause orbital evolution. Since we know that systems with multiple giant planets exist (e.g., $v$ And, Butler et al. 1999; 47 UMa, Fischer et al. 2002; HD168443, Marcy et al. 2001b; 55 Cnc, Marcy et al. 2002, and, to a lesser extent, our own), quantitative studies of these systems are needed. For some cases of multi-planet systems, planet survival statistics could be different from our results because of the additional planet-planet interactions. Additionally, if type II migration should operate significantly more or less efficiently than we have calculated here (either through the presence of another planet or some other disk-planet interaction), the overall survival and statistics we have derived here could change substantially.

\section{Conclusions}

We have presented a simple, baseline model of planet migration. Our simple model allows us to study the statistical behavior of planets migrating in disks without complications introduced from relatively unconstrained processes, like stopping mechanisms. Our overall results can be useful as a baseline statistical result of planet formation and survival. We have shown that most giant planets, under nominal initial conditions, migrate rapidly relative to the disk lifetime and are destroyed before the circumstellar disk dissipates. We have found that the observed extrasolar planets must represent only the tip of the iceberg - perhaps $25 \%$ to $33 \%$ of the total extant population has been detected - based on the required distribution of initial parameters. Most planets which have formed and survived reside outside the current detection limit of the radial velocity searches (a similar conclusion was reached by Armitage et al. 2002); this prediction is supported with new detections reported in Vogt et al. (2002). We have shown that the planetary initial mass distribution must be biased toward smaller masses in order to produce the shape of the observed EGP mass distribution, yielding our preferred initial mass distribution (see also Armitage et al. 2002); again, recent detections by Vogt et al. (2002) support the conclusion that the lowest mass planets are the most common. Finally, because migration in high mass disks destroys almost all giant planets formed therein, low mass disks - within a factor of 10 or so of the mass of Jupiter - must be capable of forming giant planets (essentially in agreement with recent observational results by Carpenter 2002). Hence we find that giant planet formation must be a relatively efficient process in disks. Because high mass planets migrate more slowly than low mass planets in a given disk, overall there should be more massive planets at intermediate and large semi-major axes than found close-in. The population of close-in planets, in contrast, should be dominated by smaller mass planets. Zucker \& Mazeh (2002) have shown that this effect is, in fact, observed and statistically significant.

Migrating giant planets may be detrimental to terrestrial planet survival, if terrestrial planets form coevally with giant planets. Planets interior to a migrating giant planet would be disrupted and lost from the system. This of course assumes that smaller planets do not migrate, although they too likely migrate, potentially on even shorter timescales than giant planets (Ward 1997a, 1997b). If terrestrial planets form after the dissipation of gas in the protoplanetary disk, then disruption by a migrating giant planet may be less of a risk (excepting giant planet migration caused by planet-planetesimal interaction, Murray et al. 1998). Kortenkamp \& Wetherill (2000) have considered the case of terrestrial planet formation when Jupiter has both its current and a larger heliocentric distance of $6.2 \mathrm{AU}$. They have found that accumulation of rocky bodies may be easier with Jupiter at a larger heliocentric distance (that is, premigration). It is possible that formation of the terrestrial planets in our Solar System may reveal clues about giant planet 
migration in our planetary system; certainly, these studies are also relevant to the formation of small, rocky planets in other planetary systems. In the context of large scale migrations, if terrestrial planet formation requires that giant planets have not migrated through the terrestrial zone around $1 \mathrm{AU}$, then only around $20 \%$ (flat initial mass distribution) or 3\% (preferred) of all planet-forming systems qualify (i.e., a few percent of all late-type stars; see Sects. 3 and 5.2). If, however, the constraint is merely that there be no giant planet in the immediate vicinity of the terrestrial planet zone at the onset of terrestrial planet formation (perhaps $10^{7}$ years), then the vast majority (93\% for flat initial mass distribution, $99 \%$ for preferred) of planet-forming systems qualify. This number corresponds to nearly all planetforming systems which is around $10 \%$ to $80 \%$ of late-type stars.

Although radial velocity detections of more distant giant planets will become possible as the time baseline of observations increases, astrometric techniques are more sensitive to giant planets in large orbits. SIM, the Space Interferometry Mission (Danner \& Unwin 1999), will do a thorough job of detecting giant planets, Uranus-mass objects, and even smaller bodies from small to large semi-major axes ( $10 \mathrm{AU})$, with maximum sensitivity achieved for planets approximately $0.3 M_{\text {Earth }}$ around 3-5 AU. SIM's ability to test our predictions of the preferred initial giant planet mass distribution will be limited largely by mission lifetime. However, SIM can also analyze disks around young stars with high precision (target resolution of 1 microarcsec), perhaps mapping out the signature of gaps created by migrating (or non-migrating) giant planets and giving us a rough snapshot of the time-dependent mass distribution of planets during the migration phase itself. (A 1 AU gap at 100 parsecs is 10 milliarcsec.) For giant planets in the largest orbits, i.e., 20-30 AU from their parent star, direct imaging techniques may be the only practical method for detection since astrometric techniques would require baselines of decades or more.

Our results suggest that techniques to study planet formation around young stars - radial velocity; high resolution imaging of young stellar systems; searches for gaps such as with SIM and potentially also the Space InfraRed Telescope Facility (SIRTF); searches for planetary outflows (Quillen \& Trilling 1998); or searches for other indirect evidence, like cometesimals scattered onto stars (Quillen \& Holman 1999) - should ultimately have a very high success rate. We anticipate that observational data will show that planet formation is taking place around $10 \%$ to $80 \%$ of low mass pre-main sequence stars, and that planet searches around main sequence stars will have a much lower success rate. Planet formation is an "easy come, easy go" business, with many planets created and many planets destroyed, and with an important minority - including our own Jupiter - surviving.

Acknowledgements. The authors thank Doug Lin, Peter Bodenheimer, Geoff Bryden, Hubert Klahr, Geoff Marcy, Fred Adams, and Norm Murray for many useful discussions. We thank an anonymous referee for useful suggestions. D.E.T. acknowledges a NASA GSRP grant, and NASA grants to Doug Lin, Peter Bodenheimer, and Robert H. Brown. J.I.L. acknowledges support from the NASA Origins Program. W.B. acknowledges support from the Swiss National Science Foundation. D.E.T. thanks E. Stein for hosting him for useful work sessions, and NASA's IRTF which accommodated him for some of the writing of this paper.

\section{References}

Armitage, P. J., Livio, M., Lubow, S. H., \& Pringle, J. E. 2002, MNRAS, 334, 248

Beckwith, S. V. W., \& Sargent, A. I. 1993, in Protostars and Protoplanets III, ed. E. H. Levy, \& J. I. Lunine (Tucson: University of Arizona Press), 521

Beckwith, S. V. W., Henning, T., \& Nakagawa, Y. 2000, in Protostars and Planets IV, ed. V. Mannings et al. (Tucson: University of Arizona), 533

Boss, A. 1997, Science, 276, 1836

Boss, A. 1998, ApJ, 503, 923

Boss, A. 2000, ApJ, 536, L101

Boss, A. 2001, ApJ, 563, 367

Bryden, G., Chen, X., Lin, D. N. C., Nelson, R. P., \& Papaloizou, J. C. B. 1999, ApJ, 514, 344

Bryden, G., Różyczka, M., Lin, D. N. C., \& Bodenheimer, P. 2000, ApJ, 540, 1091

Burrows, A., Guillot, T., Hubbard, W. B., et al. 2000, ApJ, 534, L97

Butler, R. P., \& Marcy, G. W. 1996, ApJ, 464, L153

Butler, R. P., Marcy, G. W., Williams, E., et al. 1996, PASP, 108, 500

Butler, R. P., Marcy, G. W., Williams, E., Hauser, H., \& Shirts, P. 1997, ApJ, 474, L115

Butler, R. P., Marcy, G. W., Fischer, D. A., et al. 1999, ApJ, 526, 916

Butler, R. P., Tinney, C. G., Marcy, G. W., et al. 2001, ApJ, 555, 410

Butler, R. P., Marcy, G. W., Vogt, S. S., et al. 2002, ApJ, submitted

Carpenter, J. M. 2002, AJ, 124, 1593

Charbonneau, D., Brown, T. M., Latham, D. W., \& Mayor, M. 2000, ApJ, 529, L45

Chiang, E. I., Tabachnik, S., \& Tremaine, S. 2001, AJ, 122, 1607

Cochran, W. D., Hatzes, A. P., Butler, R. P., \& Marcy, G. W. 1997, ApJ, 483, 457

D’Angelo, G., Henning, T., \& Kley, W. 2002, A\&A, 385, 647

Danner, R., \& Unwin, S. (eds.) 1999, SIM (Space Interferometry Mission): Taking the measure of the universe, NASA Jet Propulsion Laboratory, Pasadena, CA (Report \# 400-811)

Delfosse, X., Forveille, T., Mayor, M., et al. 1998, A\&A, 338, L67

Del Popolo, A., Gambera, M., \& Ercan, N. 2001, MNRAS, 325, 1402

Fischer, D. A., Marcy, G. W., Butler, R. P., Laughlin, G., \& Vogt, S. S. 2002, ApJ, 564, 1028

Gatewood, G., Han, I., \& Black, D. 2001, ApJ, 548, L61

Goldreich, P., \& Tremaine, S. 1980, ApJ, 241, 425

Greaves, J. S., Holland, W. S., Moriarty-Schieven, G., et al. 1998, ApJ, 506, L133

Guillot, T., Burrows, A., Hubbard, W. B., Lunine, J. I., \& Saumon, D. 1996, ApJ, 459, L35

Gullbring, E., Hartmann, L., Briceño, C., \& Calvet, N. 1998, 492, 323

Halbwachs, J. L., Arenou, F., Mayor, M., Udry, S., \& Queloz, D. 2000, A\&A, 355, 581

Han, I., Black, D., \& Gatewood, G. 2001, ApJ, 548, L57

Hartmann, L., Calvet, N., Gullbring, E., \& D’Alessio, P. 1998, ApJ, 495,385

Hatzes, A. P., Cochran, W. D., McArthur, B., et al. 2000, ApJ, 544, L145

Henry, G. W., Marcy, G. W., Butler, R. P., \& Vogt, S. S. 2000, ApJ, 529, L41

Kley, W. 1999, MNRAS, 303, 696

Kley, W. 2000, MNRAS, 313, L47

Kley, W., D’Angelo, G., \& Henning, T. 2001, ApJ, 547, 457

Kortenkamp, S. J., \& Wetherill, G. W. 2000, Icarus, 143, 60 
Laughlin, G., \& Chambers, J. E. 2001, ApJ, 555, L109

Lin, D. N. C., Bodenheimer, P., \& Richardson, D. C. 1996, Nature, 380, 606

Lin, D. N. C., \& Papaloizou, J. 1986, ApJ, 309, 846

Lin, D. N. C., \& Papaloizou, J. C. B. 1993, in Protostars and Protoplanets III, ed. E. H. Levy, \& J. I. Lunine (Tucson: University of Arizona Press), 749

Lin, D. N. C., Papaloizou, J. C. B., Terquem, C., Bryden, G., \& Ida, S. 2000, in Protostars and Planets IV, ed. V. Mannings, A. P. Boss, \& S. S. Russell (Tucson: University of Arizona), 1111

Lineweaver, C., \& Grether, D. 2002, Astrobiology, in press

Lissauer, J. 1987, Icarus, 69, 249

Marcy, G. W., \& Butler, P. R. 2000, PASP, 112, 137

Marcy, G. W., Butler, R. P., Vogt, S. S., Fischer, D., \& Lissauer, J. J. 1998, ApJ, 505, L147

Marcy, G. W., Butler, R. P., \& Vogt, S. S. 2000, ApJ, 536, L43

Marcy, G. W., Cochran, W., \& Mayor, M. 2000, in Protostars and Planets IV, ed. V. Mannings, A. P. Boss, \& S. S. Russell (Tucson: University of Arizona), 1285

Marcy, G. W., Butler, R. P., Fischer, D., et al. 2001a, ApJ, 556, 296

Marcy, G. W., et al. 2001b, ApJ, 555, 418

Marcy, G. W., Butler, R. P., Fischer, D. A., et al. 2002, ApJ, submitted

Mayor, M., \& Queloz, D. 1995, Nature, 378, 355

Mazeh, T., Zucker, S., dalla Torre, A., \& van Leeuwen, F. 1999, ApJ, 522, L149

McGrath, M., Nelan, E., Block, D. C., et al. 2002, ApJ, 564, L27

Murray, N., Hansen, B., Holman, M., \& Tremaine, S. 1998, Science, 279, 69

Murray, N., Paskowitz, M., \& Holman, M. 2002, ApJ, 565, 608

Nelson, R. P., Papaloizou, J. C. B., Masset, F., \& Kley, W. 2000, MNRAS, 318, 18

Noyes, R., Jha, S., Korzennik, S., Krockenberger, M., et al. 1997, ApJ, 483, L111

Papaloizou, J. C. B., \& Larwood, J. 2000, MNRAS, 315, 823

Papaloizou, J. C. B., Nelson, R. P., \& Masset, F. 2001, A\&A, 366, 263
Pepe, F., Mayor, M., Galland, F., et al. 2002, A\&A, 388, 632

Pourbaix, D. 2001, A\&A, 369, L22

Pourbaix, D., \& Arenou, F. 2001, A\&A, 372, 935

Quillen, A. C., \& Holman, M. 1999, AJ, 119, 397

Quillen, A. C., \& Trilling, D. E. 1998, ApJ, 508, 707

Santos, N. C., Mayor, M., Naef, D., et al. 2000, A\&A, 356, 599

Santos, N. C., Mayor, M., Naef, D., et al. 2001, A\&A, 379, 999

Shakura, N. I., \& Sunyaev, R. A. 1973, A\&A, 24, 337

Stevenson, D. J., \& Lunine, J. I. 1988, Icarus, 75, 146

Takeuchi, T., Miyama, S. M., \& Lin, D. N. C. 1996, ApJ, 460, 832

Tanaka, H., Takeuchi, T., \& Ward, W. R. 2002, ApJ, 565, 1257

Thommes, E. W., Duncan, M. J., \& Levison, H. F. 1999, Nature, 402, 635

Trilling, D. E. 2000, ApJ, 537, L61

Trilling, D. E., Benz, W., Guillot, T., et al. 1998, ApJ, 500, 428

Trilling, D. E., \& Brown, R. H. 1998, Nature, 395, 775

Trilling, D. E., Brown, R. H., \& Rivkin, A. S. 2000, ApJ, 529, 499

Vogt, S. S., Marcy, G. W., Butler, R. P., \& Apps, K. 2000, ApJ, 536, 902

Vogt, S. S., Butler, R. P., Marcy, G. W., et al. 2002, ApJ, 568, 352

Ward, W. R. 1997a, ApJ, 482, L211

Ward, W. R. 1997b, Icarus, 126, 126

Ward, W. R., \& Hahn, J. M. 2000, in Protostars and Planets IV, ed. V. Mannings, A. P. Boss, \& S. S. Russell (Tucson: University of Arizona), 1135

Ward, W. R., \& Hourigan, K. 1989, ApJ, 347, 490

Weidenschilling, S. L., \& Marzari, F. 1996, Nature, 384, 619

Wuchterl, G., Guillot, T., \& Lissauer, J. J. 2000, in Protostars and Planets IV, ed. V. Mannings, A. P. Boss, \& S. S. Russell (Tucson: University of Arizona), 1081

Zucker, S., \& Mazeh, T. 2000, ApJ, 531, L67

Zucker, S., \& Mazeh, T. 2001a, ApJ, 562, 549

Zucker, S., \& Mazeh, T. 2001b, ApJ, 562, 1038

Zucker, S., \& Mazeh, T. 2002, ApJ, 568, L113

Zuckerman, B., Forveille, \& Kastner, J. H. 1995, Nature, 373, 494 